\title{
低价(正一价)镁金属化合物的合成及其应用研究进展
}

\author{
马猛涛 $*, a$ 王未凡 ${ }^{a}$ 姚薇薇 $*, b$ \\ ( ${ }^{a}$ 南京林业大学理学院 南京 210037) \\ ${ }^{b}$ 南京中医药大学药学院 南京 210023)
}

\begin{abstract}
摘要 第二主族的碱土金属价态通常为 0 价和 +2 价. 2007 年, 室温条件下稳定的一价镁金属化合物首次被成功合成, 随后一系列不同有机配体稳定的一价镁化合物陆续被制备，它们已被证实在有机反应和无机反应中可作为可溶的、化 学计量可控的、具有选择性的和安全的还原剂替代一些传统的还原剂. 主要介绍迄今为止室温稳定的一价镁金属化合 物的制备方法以及其在无机、有机化学等方面的应用的最新进展.
\end{abstract}

关键词 金属-金属键; 一价; 镁金属; 合成; 应用

\section{Synthesis of Magnesium(I) Complexes and Their Applications}

\author{
Ma, Mengtao ${ }^{*, a}$ Wang, Weifan ${ }^{a} \quad$ Yao, Weiwei*,b \\ ( ${ }^{a}$ College of Science, Nanjing Forestry University, Nanjing 210037) \\ ( ${ }^{b}$ College of Pharmacy, Nanjing University of Chinese Medicine, Nanjing 210023)
}

\begin{abstract}
The valence of the alkaline-earth metals (group 2 metals) usually is 0 and +2 . In 2007, the first room temperature stable magnesium(I) complexes were synthesized. Subsequently a series of different organic ligands stablized Mg(I) complexes have been prepared. They can be used as hydrocarbon soluble, stoichiometric, selective, and safe reducing agents in organic and inorganic reactions to replace some traditional reductants. In this review the synthesis of room temperature stable magnesium(I) complexes and their applications in organic and inorganic chemistry are briefly introduced.
\end{abstract}

Keywords metal-metal bond; univalence; magnesium; synthesis; applications

化学元素周期表中最主要的元素是金属元素, 周期 表中的 $d$ 区、 $f$ 区、 $s$ 区(除氢、氦以外)和 $\mathrm{p}$ 区的大部分 元素都是金属或类金属元素. 近几十年来, 科学家一直 对金属-金属键的研究十分感兴趣, 了解到更多金属-金 属键的性质. 最早发现的是 $\mathrm{d}$ 区和 $\mathrm{p}$ 区的金属一金属键, 最重要的发现是 50 多年前的 $\mathrm{K}_{2}\left[\mathrm{Re}_{2} \mathrm{Cl}_{8}\right] \cdot 2 \mathrm{H}_{2} \mathrm{O}$ 的合成, 这是由 Cotton 等 ${ }^{[1]}$ 首次报道的具有金属-金属四重键化 合物. $d$ 区金属键近期两个最具代表性的例子是 2004 年 Carmona 等 ${ }^{[2]}$ 在 Science 期刊上报道的一价锌二聚物, $\mathrm{Cp}^{*} \mathrm{Zn}-\mathrm{ZnCp} *\left(\mathrm{Cp}^{*}=\mathrm{C}_{5} \mathrm{Me}_{5}\right)$ 和 2005 年 Power 等 ${ }^{[3]}$ 在 Science 期刊上报道的 $\mathrm{Cr}-\mathrm{Cr}$ 五重键螯合的化合物 $\mathrm{Ar}^{*} \mathrm{CrCrAr}^{*}$ (Ar*=空间位阻较大的三联苯)的合成. $\mathrm{p}$ 区 金属键的研究取得重大突破, 主要是合成了许多含多重 键的第 $13 \sim 15$ 族金属-金属化合物 ${ }^{[4]}$. 最典型的是由
Power, Sekiguchi, Wiberg 等 ${ }^{[5 \sim 10]}$ 分别制备的第 14 族炔的 类似物 REER $(\mathrm{E}=\mathrm{Si}, \mathrm{Ge}, \mathrm{Sn}, \mathrm{Pb} ; \mathrm{R}=$ 位阻较大的取代 基). 两个 $\mathrm{f}$ 区的金属能否成键并不知晓，但是在过去的 十年里，一个 $\mathrm{f}$ 区金属和一个 $\mathrm{d}$ 区或 $\mathrm{p}$ 区金属成键已有 报道.

两个 $\mathrm{s}$ 区金属能否成键? 各种理论研究表明, HMg$\mathrm{MgH}$ 或 $\mathrm{ClMgMgCl}$ 等化合物可以存在, 实验也证明了 这些化合物可以被制备, 但需要在极低的反应温度, 如 上述化合物只有在 $10 \mathrm{~K}$ 左右的低温下才能合成和研 究 ${ }^{[11,12]}$. 在 $R M M R$ 化合物中存在着 $M-M$ 键 $(R=$ 阴离 子配体, $\mathrm{M}=$ 第 2 主族金属), 它们的 $\mathrm{M}-\mathrm{M}$ 键较强(尤其 是 $M=B e$ 或 $M g$ ), 但只有具有一定空间位阻的配体才 有足够的动力来稳定 $\mathrm{M}-\mathrm{M}$ 键. 2007 年 Jones 和 Stasch 等 ${ }^{[13]}$ 在 Science 期刊上报道首次以胍基和 $\beta$-二亚胺配体

*E-mail:mengtao@njfu.edu.cn; yww0715@hotmail.com

Received July 16, 2015; revised September 12, 2015; published online September 17, 2015

Project supported by the National Natural Science Foundation of China (No. 21372117), the Natural Science Foundation of Jiangsu Province (Nos. BK20141468, BK20130952) and the Qing Lan Project of Jiangsu Province.

国家自然科学基金(No. 21372117)、江苏省自然科学基金(Nos. BK20141468, BK20130952)和江苏省 “青蓝工程” 资助项目. 
合成了相应的稳定的一价镁化合物 [\{(Priso) $\left.\mathrm{Mg}\}_{2}\right](1)$ 和 [\{( $\left.\left.\left.{ }^{\mathrm{Dipp}} \mathrm{Nacnac}\right) \mathrm{Mg}\right\}_{2}\right]$ (2). 随后选择不同空间位阻的 $\beta$-二 亚胺配体合成了一系列相应的一价镁化合物 ${ }^{[14]}$, 它们具 有很高的热稳定性, 溶于大多数有机溶剂, 对空气和湿 度不是特别敏感, 相对安全 (与碱金属钠和钾等相比无 火灾隐患)等优点. 上述一价镁化合物在无机、金属有机 和有机反应中是很好的还原剂, 还原能力中等, 在某些 方面甚至可以代替传统的还原剂, 应用于许多合成反应 ${ }^{[15,16]}$. 本文主要根据最近的研究进展以及结合我们的研 究工作, 着重介绍一价镁金属化合物的合成方法和结构 特点, 以及其在无机、金属有机和有机等反应中的应用.

\section{1 一价镁(和锌)金属化合物的合成}

\section{1 一价锌化合物的合成}

一价录很早就被人们熟知, 然而同族的一价锌化合 物直到 2004 年才被西班牙的 Carmona 等 ${ }^{[2]}$ 在一次偶然 的实验中发现, 他们用五甲基环戊二烯基 $\left(\mathrm{Cp}^{*}\right)$ 二价锌 化合物 $\left[\mathrm{Zn}\left(\mathrm{Cp}^{*}\right)_{2}\right]$ 和二乙基锌 $\left(\mathrm{ZnEt}_{2}\right)$ 反应意外地得到了 稳定一价锌化合物 $\left[\mathrm{Zn}_{2}\left(\eta^{5}-\mathrm{Cp}^{*}\right)_{2}\right]$ (图 1$)$, 然后用 $\left[\mathrm{Zn}\left(\mathrm{Cp}^{*}\right)_{2}\right]$ 、氯化锌和氢化钾以 $1: 1: 2$ 的物质的量比, 以较方便的方法高产率地得到一价锌化合物 $\left[\mathrm{Zn}_{2}\left(\eta^{5}-\mathrm{Cp}^{*}\right)_{2}\right]$. 令人惊奇的是当五甲基环戊二烯基变换 为其它类似的茂基 $\left(\mathrm{Cp}^{\prime}=\mathrm{C}_{5} \mathrm{H}_{5}, \mathrm{C}_{5} \mathrm{Me}_{4} \mathrm{H}, \mathrm{C}_{5} \mathrm{Me}_{4} \mathrm{SiMe}_{3}\right.$, $\left.\mathrm{C}_{5} \mathrm{Me}_{4} \mathrm{Bu}-t\right)$ 时，仅有四甲基乙基取代的茂基 $\left(\mathrm{Cp}^{\prime}=\right.$ $\left.\mathrm{C}_{5} \mathrm{Me}_{4} \mathrm{Et}\right)$ 得到相应的一价锌化合物 $\left[\mathrm{Zn}_{2}\left(\eta^{5}-\mathrm{C}_{5} \mathrm{Me}_{4} \mathrm{Et}\right)_{2}\right]$, 这意味着茂基上的取代基的细微变化对一价锌化合物 的稳定性和形成具有显著的影响 ${ }^{[17]}$. 这一突破性的发 现立刻引起了其他研究人员浓厚的兴趣, 很快就用各种 不同类型的配体合成出相对应的一价锌金属化合物 ${ }^{[18,19]}$. 其中 Yang 课题组 ${ }^{[20 ~ 24]}$ 利用 $\alpha$-二亚胺配体 ${ }^{\mathrm{MeDipp}} \mathrm{DAB}\left({ }^{\mathrm{MeDipp}} \mathrm{DAB}=\left[(\mathrm{DippNCMe})_{2}\right], \mathrm{Dipp}=2,6\right.$-二异 丙基苯基)成功地合成了一系列含有钠或钾离子的一价 锌金属有机化合物.

在成功合成上述一价锌化合物后, 研究人员随后考 察了它们的反应性能, 其中研究最多的是 $\left[\mathrm{Zn}_{2}\left(\eta^{5}-\mathrm{Cp}^{*}\right)_{2}\right]$. 起初发现 $\left[\mathrm{Zn}_{2}\left(\eta^{5}-\mathrm{Cp}^{*}\right)_{2}\right]$ 并不能与氢气、 一氧化碳和二氧化碳等小分子和常见的含氮或膦的路 易斯碱(如三乙胺、吡啶和三苯基膦等)反应, 进一步的 研究显示它能与强的路易斯碱[如 dmap (4-dimethylaminopyridine), pyr-py (4-pyrrolidinopyridine), DBU (1,8-diazabicyclo[5.4.0]undec-7-ene)]形成路易斯酸碱加 合物, 与后过渡金属反应生成新颖的含有独特单电子配 体 $\mathrm{Cp} * \mathrm{Zn}$ 和 $\mathrm{Cp} * \mathrm{Zn}_{2}$ 的金属间配合物, 这为簇合物的合 成开启了新的途径. 最近 Roesky 等 ${ }^{[25]}$ 首次发现 $\left[\mathrm{Zn}_{2}\right.$ $\left.\left(\eta^{5}-\mathrm{Cp}^{*}\right)_{2}\right]$ 以 $2.5 \mathrm{~mol} \%$ 比作为均相催化剂高效催化苯胺
和㶧的氢氨化反应.

\section{2 一价镁化合物的合成}

通常碱金属的氧化态主要是 +1 价, 碱土金属的氧 化态主要是 +2 价, 碱土金属的氧化还原性非常稳定. 由于镁和锌在很多方面具有一定的相似性，比如它们的 原子半径基本相同(锌, $142 \mathrm{pm}$; 镁, $145 \mathrm{pm}$ ), 最外层电 子都只有 2 个价电子. 受到一价锌化合物成功合成的启 发, 同时量子化学计算模拟显示类似的一价镁也可以稳 定存在, 2007 年澳大利亚的 Jones 和 Stasch 等 ${ }^{[13,14]}$ 用胍 基配体 Priso $\left[\mathrm{Priso}=(\mathrm{DippN})_{2} \mathrm{CN}(i-\mathrm{Pr})_{2}\right]$ 和 $\beta$-二亚胺配体 ${ }^{\text {Dipp }} \mathrm{NacnacH}\left[{ }^{\text {Dipp }} \mathrm{Nacnac}=\left({ }^{\text {Dipp }} \mathrm{NCMe}\right)_{2} \mathrm{CH}^{-}\right]$分别与格氏 试剂 MeMgI 反应, 生成相应的二价镁碘化物前体 $\left[\{(\mathrm{Priso}) \mathrm{MgI}\}_{2}\left(\mathrm{OEt}_{2}\right)\right]$ 和 $\left.\left[{ }^{\mathrm{Dipp}} \mathrm{Nacnac}\right) \mathrm{MgI}\left(\mathrm{OEt}_{2}\right)\right]$, 然后室 温条件下用过量的钾还原, 首次成功地合成了室温条件 下稳定的一价镁化合物 [ $\left\{\right.$ (Priso) $\left.\mathrm{Mg}_{2}\right]$ (1, 无色) 和 [ $\left\{\left({ }^{\text {Dipp }} \mathrm{Nacnac}\right) \mathrm{Mg}_{2}\right]$ (2, 黄色 $)$, 然后通过同样的合成方 法制备出一系列 $\beta$-二亚胺配体稳定的一价镁金属化合 物 $\left.\left[\left\{{ }^{\mathrm{Ar}} \mathrm{Nacnac}\right) \mathrm{Mg}\right\}_{2}\right](\mathbf{3} \sim \mathbf{5})$ (Scheme 1).

空间位阻较大的叔丁基取代的 $\beta$-二亚胺配体 ${ }^{t-\mathrm{Bu}} \mathrm{Na}-$ $\mathrm{cnacH}$ 与格氏试剂不能发生上述类似的反应生成相应的 二价镁的碘化物前体，因此必须用其它的合成方法，即 ${ }^{t-\mathrm{Bu}} \mathrm{NacnacH}$ 配体和二丁基镁在甲苯中反应 $16 \mathrm{~h}$, 原位生 成 $\left[\left(^{t-\mathrm{Bu}} \mathrm{Nacnac}\right) \mathrm{Mg}{ }^{n} \mathrm{Bu}\right]$, 然后再加入等物质的量的单质 碘, 得到相应的碘化镁前体 $\left[\left(^{t-\mathrm{Bu}} \mathrm{Nacnac}\right) \mathrm{MgI}\right]$, 最后用金 属钾还原得到较大空间位阻的一价镁化合物 $\left.\left[\left\{c^{t-\mathrm{Bu}} \mathrm{Nacnac}\right) \mathrm{Mg}\right\}_{2}\right] \quad\left(\mathbf{6},{ }^{t-\mathrm{Bu}} \mathrm{Nacnac}=\left[{ }^{\mathrm{Dipp}} \mathrm{NC}(t-\mathrm{Bu})\right]_{2} \mathrm{C}-\right.$ $\mathrm{H})^{[14]}$. 尝试用类似一价镁化合物的合成方法制备稳定 的一价铍和钙化合物, 至今没有获得成功, 包括 $\left({ }^{\mathrm{Mes}} \mathrm{Nacnac}\right) \mathrm{BeI}$ 和 $\left[\left({ }^{\mathrm{Dipp}} \mathrm{Nacnac}\right) \mathrm{CaI}\left(\mathrm{OEt}_{2}\right)\right]$ 的碱金属还原, 均得到了复杂的混合物.

空间位阻稍小的苯基取代的 $\left[\left({ }^{\mathrm{Ph}} \mathrm{Nacnac}\right) \mathrm{MgI}\left(\mathrm{OEt}_{2}\right)\right]$ $\left({ }^{\mathrm{Ph}} \mathrm{Nacnac}=\left[(\mathrm{PhNCMe})_{2} \mathrm{CH}\right]^{-}\right)$的还原不能得到相应的一 价镁化合物, 而是得到均聚的二价镁化合物 $\left[{ }^{\mathrm{Ph}} \mathrm{Nacn}\right.$ ac $)_{2} \mathrm{Mg}$ ], 这表明苯基取代的 $\beta$-二亚胺配体没有足够大 的空间体积来稳定活泼的一价镁化合物, 以防止其歧化 反应. 另一方面配体的空间位阻过大同样也不能得到相 应的一价镁金属化合物, 例如当 $\beta$-二亚胺配体 ${ }^{\mathrm{Dipp}} \mathrm{Nacn}$ $\mathrm{acH}$ 中的 2,6-二异丙基被位阻更大的二苯甲基 $\left(\mathrm{Ph}_{2} \mathrm{CH}\right)$ 取代或苯的对位引入二苯甲基 $\left(\mathrm{Ph}_{2} \mathrm{CH}\right)$ 或三苯甲基 $\left(\mathrm{Ph}_{3} \mathrm{C}\right)$ 时, 目前为止, 我们暂时还未能分离出相应的一 价镁化合物，均得到了原料或配体碱金属盐. 反应溶剂 和还原试剂对一价镁化合物的制备也有很大的影响，例 如 $\left[\left({ }^{\text {Dipp }} \mathrm{Nacnac}\right) \mathrm{MgI}(\mathrm{THF})\right]$ 的四氢呋喃络合物虽然能与 钠镜反应, 但产率非常低, 而 $\left.\left[{ }^{\mathrm{Dipp}} \mathrm{Nacnac}\right) \mathrm{MgI}\left(\mathrm{OEt}_{2}\right)\right]$ 的 还原用乙醚和甲苯作为溶剂则可以得到 $50 \%$ 左右的产 


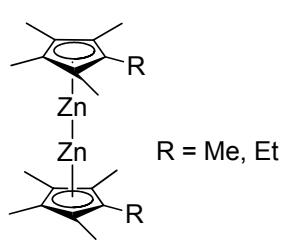

Carmona et al. 2004

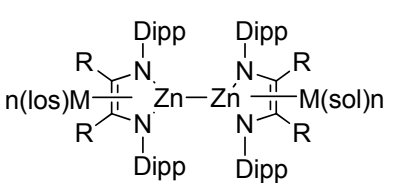

$\mathrm{R}=\mathrm{H}, \mathrm{Me} ; \mathrm{M}=\mathrm{Na}, \mathrm{K} ; \mathrm{sol}=\mathrm{THF}, \mathrm{Et} 2 \mathrm{O}$

Yang, X. J. et al. $2007 \sim 2012$

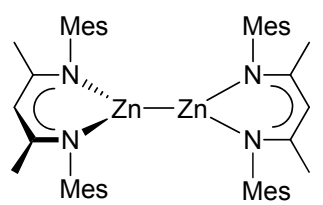

Schulz et al. 2009

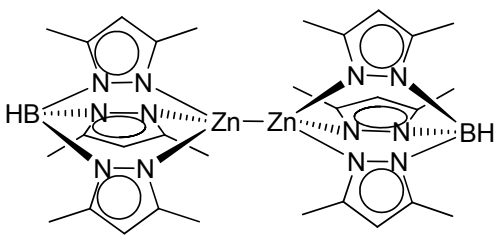

Schulz et al. 2010

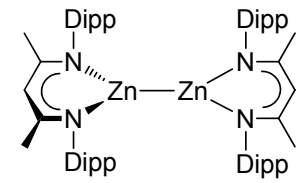

Robinson et al. 2005

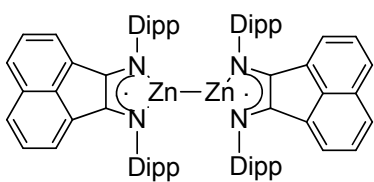

Fedushkin et al. 2007

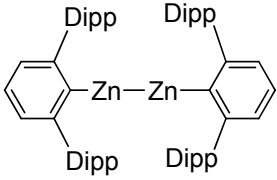

Power et al. 2006

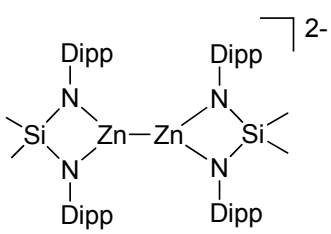

Tsai et al. 2007

Schulz et al. 2010<smiles></smiles>

Roesky et al. 2011

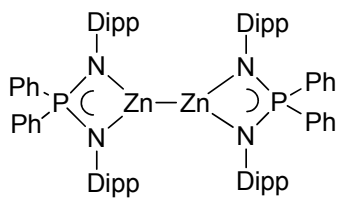

Stasch 2012

图 1 一价锌金属有机化合物

Figure 1 Zinc(I) organometallic complexes
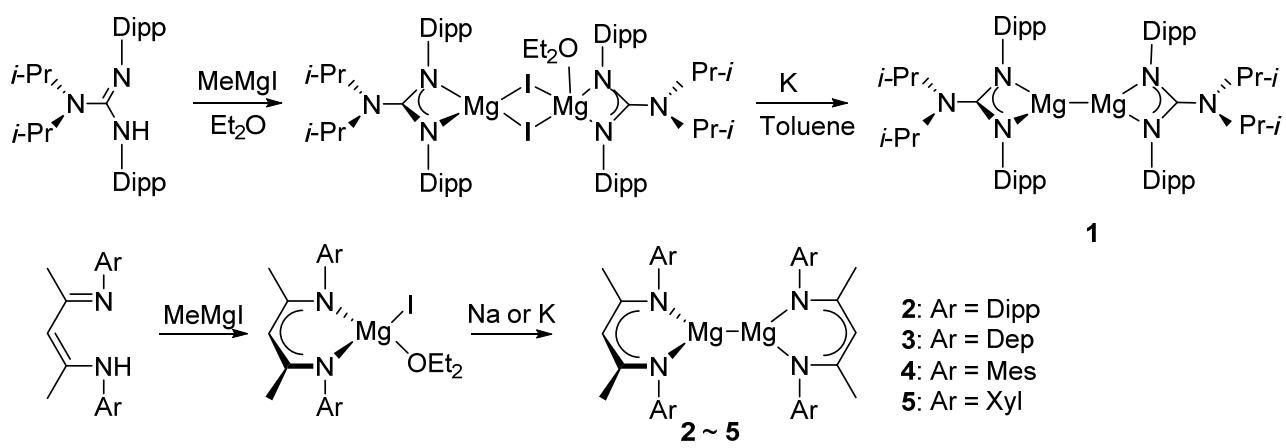<smiles>CC(C)c1cccc(C(C)C)c1N</smiles>

( Dipp )<smiles>CCc1cccc(CC)c1N</smiles>

(Dep )<smiles>Cc1cc(C)c(C)c(C)c1C</smiles>

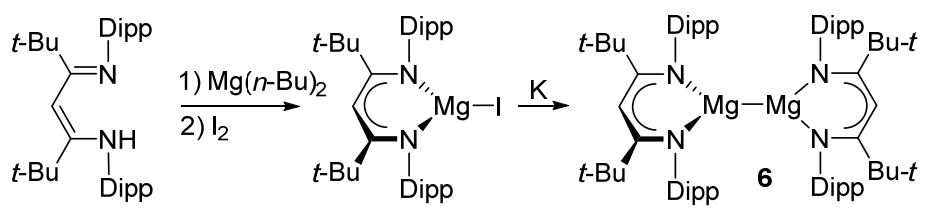

图式 1 一价镁金属化合物 1 6 的合成

Scheme 1 Synthesis of $\mathrm{Mg}(\mathrm{I})$ complexes $\mathbf{1} \sim \mathbf{6}$ 
率, $\left[\left({ }^{\mathrm{Dipp}} \mathrm{Nacnac}\right) \mathrm{MgI}\left(\mathrm{OEt}_{2}\right)\right]$ 的还原反应如果用金属钠作 为还原剂需要 $5 \sim 7 \mathrm{~d}$, 而用钾作为还原剂仅需要 $1 \mathrm{~d}$, 如 果反应过长, 产率反而降低, 可能由于过度还原的原因.

Jones 等 ${ }^{[14]}$ 通过碱金属还原 $1: 1$ 比例的两种不同的 二价镁碘化物前体, 希望制备出混合 $\beta$-二亚胺配体稳定 的一价镁化合物, 然而反应通常产生混合物, 且没有得 到目标产物。例如钾还原二价镁的碘化物前体 $\left[\left({ }^{\mathrm{Dipp}} \mathrm{Nacnac}\right) \mathrm{MgI}\left(\mathrm{OEt}_{2}\right)\right]$ 和 $\left.\left[{ }^{\mathrm{Mes}} \mathrm{Nacnac}\right) \mathrm{MgI}\left(\mathrm{OEt}_{2}\right)\right]$ 的 1 : 1 混合物, 得到产率较低的混合氢/碘桥连产物 $\left[\left({ }^{\mathrm{Dipp}} \mathrm{Nacnac}\right) \mathrm{Mg}(\mu-\mathrm{H})(\mu-\mathrm{I}) \mathrm{Mg}\left({ }^{\mathrm{Mes}} \mathrm{Nacnac}\right)\right]$. 推测氢化物 可能来源于溶剂提取过程. 然后他们重新检测了 $\left[\left({ }^{\mathrm{Dipp}} \mathrm{Nacnac}\right) \mathrm{MgI}\left(\mathrm{OEt}_{2}\right)\right]$ 的钾还原反应, 核磁分析表明除 主要产物一价镁化合物 2 , 还有约 $15 \%$ 的二价镁氢化物 $\left[\left\{\left(^{\mathrm{Dipp}} \mathrm{Nacnac}\right) \operatorname{Mg}(\mu-\mathrm{H})\right\}_{2}\right]$ 和混合氢/碘桥连产物 $\left[\left({ }^{\text {Dipp }} \mathrm{Nacnac}\right) \operatorname{Mg}(\mu-\mathrm{H})(\mu-\mathrm{I}) \mathrm{Mg}\left(^{\text {Dipp }} \mathrm{Nacnac}\right)\right]$ 组成的混合物. 他们推测从二价镁的碘化物 $\left[\left({ }^{\mathrm{Ar}} \mathrm{Nacnac}\right) \mathrm{MgI}\left(\mathrm{OEt}_{2}\right)\right]$ 到最 终一价镁化合物 [\{( $\left.\left.\left.{ }^{\mathrm{Ar}} \mathrm{Nacnac}\right) \mathrm{Mg}\right\}_{2}\right]$ 的合成可能经过中间 镁的氢化物 $\left[\left\{\left({ }^{\mathrm{Ar}} \mathrm{Nacnac}\right) \mathrm{Mg}(\mu-\mathrm{H})\right\}_{2}\right]$ 这一过程, 后来的实 验证明了该推测, 但产率较低.

前面提到溶剂对还原反应有较大的影响, 2009 年我 国 Yang 课题组 ${ }^{[26]}$ 将与 $\beta$-二亚胺结构类似的 $\alpha$-二亚胺配 体 ${ }^{\mathrm{MeDipp}} \mathrm{DAB}$ 、氯化镁和金属钾在四氢呋喃中用 “一锅 法” 成功合成出 $\alpha$-二亚胺配体稳定的一价镁化合物 $\left[\left\{\left[\mathrm{K}(\mathrm{THF})_{3}\right]\left({ }^{\mathrm{MeDipp}} \mathrm{DAB}\right) \mathrm{Mg}_{2}\right]\right.$ (7, 粉红色), 与 $\beta$-二亚胺 配体不同, 溶剂四氢呋喃对 $\alpha$-二亚胺配位的一价镁化合 物的合成没有影响(Eq. 1). 然而还原剂钾的用量对产物 的合成有较大的影响, 当 $\alpha$-二亚胺配体与钾的物质的量 比为 $1: 1$ 时, 均得到了二价镁的均聚物 ( $\mathrm{LMgL}, \mathrm{L}=$ 配 体); 当物质的量比为 $1: 2$ 时, 产物依然是二价镁化合
物 $\operatorname{LMg}(\mathrm{THF})_{n}$, 不同的是只有一个配体与镁金属配位, 其它配位位置为四氢呋喃，这与另一份钾金属继续反应 可以得到一价镁化合物; 当物质的量比为 $1: 3$ 时, 空间 位阻较大的 2,6-二异丙基苯基取代的 $\alpha$-二亚胺顺利分离 出目标产物一价镁化合物，而空间位阻稍小的 2,4,6-三 甲基苯基取代的 $\alpha$-二亚胺则得到含钾离子的二价镁化 合物 ${ }^{[27]}$.

2014 年, $\mathrm{Stasch}^{[28]}$ 将膦亚胺配体 $\mathrm{Ph}_{2} \mathrm{P}(\mathrm{NDipp})_{2} \mathrm{H}$ 和 格氏试剂 $\mathrm{MeMgX}(\mathrm{X}=\mathrm{Br}$ or $\mathrm{I})$ 在乙醚中反应得到二价 镁卤化物 $\left[\mathrm{LMgX}\left(\mathrm{OEt}_{2}\right)\right]$ 前体, 化合物 $\left[\mathrm{LMgX}\left(\mathrm{OEt}_{2}\right)\right]$ 中配 位溶剂乙醚可以非常容易除去, 只须将其用甲苯重新溶 解, 然后加热降压即可除去, 得到非溶剂的二聚物 $\left[(\mathrm{LMgX})_{2}\right]$. 如果用钠或钾金属还原上述化合物 $\left[(\mathrm{LMgX})_{2}\right]$, 不能得到一价镁化合物, 只能分离出配体 的碱金属盐如 [LK]. 这与相同配体二价锌化合物 $\left[(\mathrm{LZnX})_{2}\right]$ 的还原类似, 不能得到相应的一价锌化合物, 这可能由于用 $\mathrm{Na}, \mathrm{K}$ 或 $\mathrm{KC}_{8}$ 作为还原剂时，它们会被过 度还原而产生混合物。但如果用前面提到的一价镁 $\left[\left\{\left({ }^{\mathrm{Mes}} \mathrm{Nacnac}\right) \mathrm{Mg}\right\}_{2}\right](4)$ 作为还原剂, 则能成功分离出目 标产物. 一价镁化合物 4 和前体 $\left[(\operatorname{LMgX})_{2}\right]$ 在 $80{ }^{\circ} \mathrm{C}$ 甲苯 或苯中反应 $1 \mathrm{~h}$ 左右顺利得到新的无色一价镁化合物 $\mathbf{8}$, 产率高达 $60 \%$ 以上(Scheme 2). 令人惊奇的是用结构类 似的一价镁 2 作还原剂则不能得到 8. 室温或低温条件 下用 4 还原溶剂化的 $\left[\mathrm{LMgX}\left(\mathrm{OEt}_{2}\right)\right]$ 时, 通过核磁谱图分 析均得到了各种各样的混合物也包括 4 . 反应时溶液颜 色变化很快, 由红棕色很快变为深棕色, 最后变为黑色 金属镁沉淀，这些现象与最近合成的亚稳态的 “ $\mathrm{Mg}^{\mathrm{I}} \mathrm{Br}$ ” 棕色溶液相似 ${ }^{[29 ~ 31]}$, 即使在低温下也会歧化.

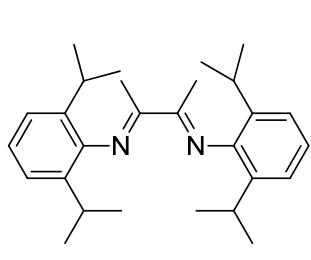

(2) $\mathrm{MgCl}_{2}$
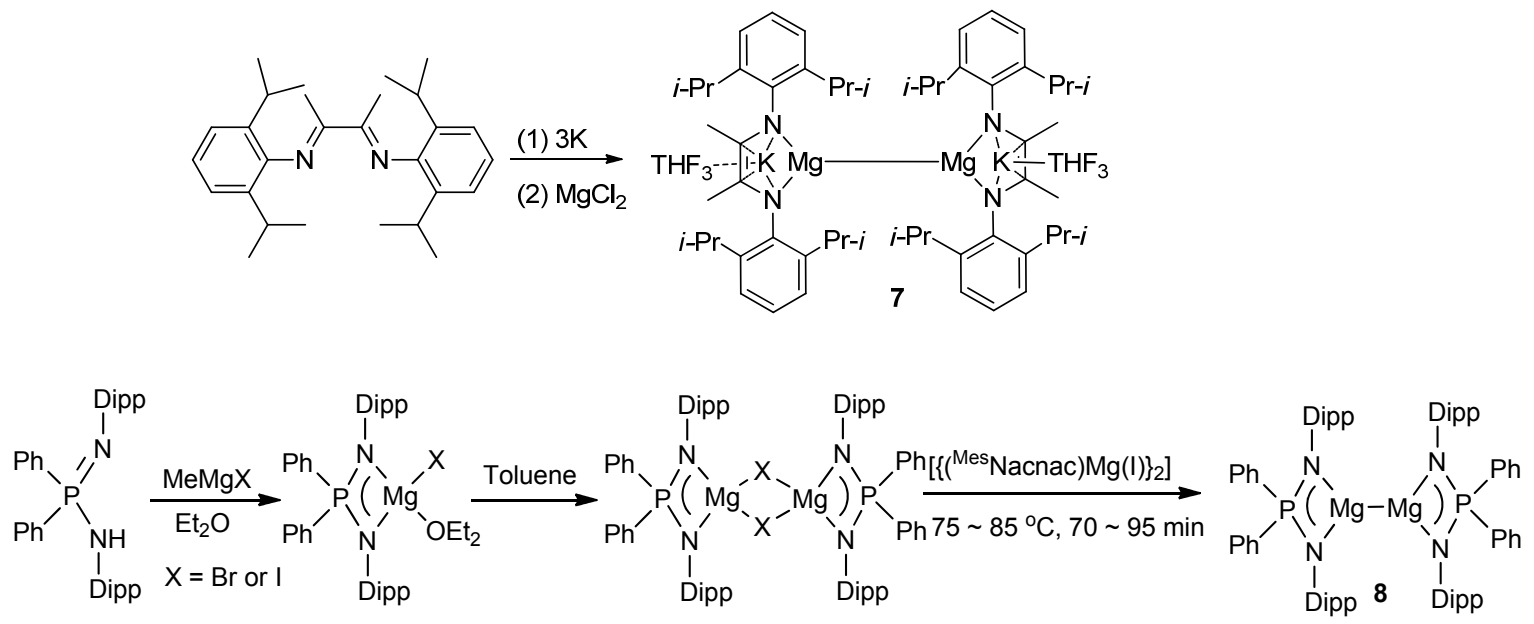

图式 2 一价镁金属化合物 8 的合成

Scheme 2 Synthesis of $\mathrm{Mg}(\mathrm{I})$ complex 8 


\section{2 一价镁金属化合物的应用}

众所周知金属镁和含镁的化合物已经被应用 100 多 年, 其中格氏试剂是化学中被广泛应用的化学试剂之 一, 特别是在有机合成中被大量应用于各种各样的有机 反应中, 合成出众多的新化合物, 现已得到了商品化. 与零价金属镁和二价镁化合物的悠久历史相比, 一价镁 化合物由于最近才被合成出来, 它的应用相对较少. 一 旦室温条件下稳定的一价镁化合物可重复使用, 就有机 会进一步探讨这些活性物质的化学反应. 这些活性物质 在无机、金属有机和有机反应中是很好的还原剂, 在某 些方面甚至可以代替相对传统的还原剂(如镁金属、碱 金属和二碘化钐等)，应用于许多合成反应. 虽然没有 数据表明一价镁化合物的还原能力, 但是它的还原能力 应该在二碘化钐和碱金属之间 ${ }^{[32 \sim 34]}$. 如果这样, 一价镁 化合物应当被归类为中性至强性的还原剂. 迄今为止, 一价镁化合物应用最多的是 $\beta$-二亚胺配体稳定的一价 镁 $\left[\left\{\left({ }^{\mathrm{Mes}} \mathrm{Nacnac}\right) \mathrm{Mg}_{2}\right]\right.$ (4) 和 $\left[\left\{\left({ }^{\mathrm{Dipp}} \mathrm{Nacnac}\right) \mathrm{Mg}\right\}_{2}\right]$ (2), 因 为它们比胍基配位的一价镁化合物 [ $\left.\{\text { (Priso) } M g\}_{2}\right]$ (1)产 率高, 热稳定性好, 绝大部分的研究工作主要是由 Jones 和 Andreas 课题组以及他们的合作者完成的.

\section{1 一价镁化合物在金属有机和无机合成中的应用}

在过去的数十年里, 有关 $\mathrm{p}$ 区低氧化态等方面的研 究得到迅速发展, 不仅是因为对这类化合物的基础知识 感兴趣, 更是因为它们在合成和应用方面的高反应活 性 ${ }^{[35 ~ 37]}$. 在此领域中, 能否找到合适的还原剂还原正常 氧化态的前体化合物至关重要. 目前最广泛使用的还原 剂是 $\mathrm{s}$ 区的金属(通常是锂、钠、钾或镁等金属)、石墨 钾 $\left(\mathrm{KC}_{8}\right)$ 和菜碱金属化合物, 然而这些还原剂经常会导 致过度还原和产生副反应等问题, 这主要是因为这些试 剂还原性太强和溶解性差. 这两个因素使得难以控制还
原剂的用量和反应如何选择哪种还原剂，此外由于碱金 属等反应活性太高，非常容易引起实验室火灾，处理需 要十分小心. 为了克服传统还原剂的上述缺点, 在成功 合成一价镁化合物之后, Jones 和 Andreas 以及其他研究 人员开始探索一价镁化合物的应用，发现一价镁化合物 可溶于大部分有机溶剂，可以控制反应的用量及反应选 择性的，并且是相对安全的还原剂. 现将一价镁化合物 近年来在无机和金属有机合成方面的应用总结如下.

\section{1 .1 一价镁化合物在 13 主族中的应用}

最初 Jones 等将一价镁化合物和氢气直接反应，希 望得到相应二价镁的氢化物 $\left[\mathrm{LMg}(\mu-\mathrm{H})_{2} \mathrm{MgL}\right](\mathrm{L}=$ 胍基 或 $\beta$-二亚胺), 因为 $\mathrm{Mg} / \mathrm{MgH}_{2}$ 是重要的储氢系统. 计算 机模拟研究显示胍基配位的一价镁化合物和氢气反应 理论上应该是自发的，因为这类反应是放热反应(104.5 $\mathrm{J} / \mathrm{mol}$ ). 但实际上 1 和相关的 $\beta$-二亚胺配位的一价镁化 合物，即使在 $80{ }^{\circ} \mathrm{C} 、 1$ 个或 5 个标准大气压下，和氢气 也不反应. 虽然化合物 2 或 4 在 $80{ }^{\circ} \mathrm{C} 、 70$ 标准大气压 下能与氢气反应，但反应得到的产物为混合物且不包括 相应的二价镁氢化物, 这可能是由于反应动能能垒阻碍 一价镁化合物在正常条件下与氢气发生反应. 进一步研 究发现三价铝氢化物作为氢源, 可以与一价镁化合物 2 和 4 发生反应，生成相应的二价镁的氢化物，而同时产 生的副产物 10,12 是首次室温条件下稳定存在的中性二 价铝氢化物(Scheme 3$)^{[38]}$. 化合物 $\mathbf{1 0}\left(190{ }^{\circ} \mathrm{C}\right)$ 尤为重要, 因为这是 $\mathrm{Al}_{2} \mathrm{H}_{4}$ 的卡宾加合物, 先前只有在温度约 $5 \mathrm{~K}$ 时 才可以观察到 ${ }^{[39]}$. 毫无疑问, 化合物 $\mathbf{1 0}$ 的热稳定性与 其空间体积和卡宾配体的亲核性密切相关. 此外, 成功 合成化合物 10 和 12 的亮点是一价镁化合物可作为化学 选择性的还原剂, 之前尝试使用其他试剂比如荎基钠, 都不能还原 13 主族金属氢化物. 密度泛函理论(DFT)
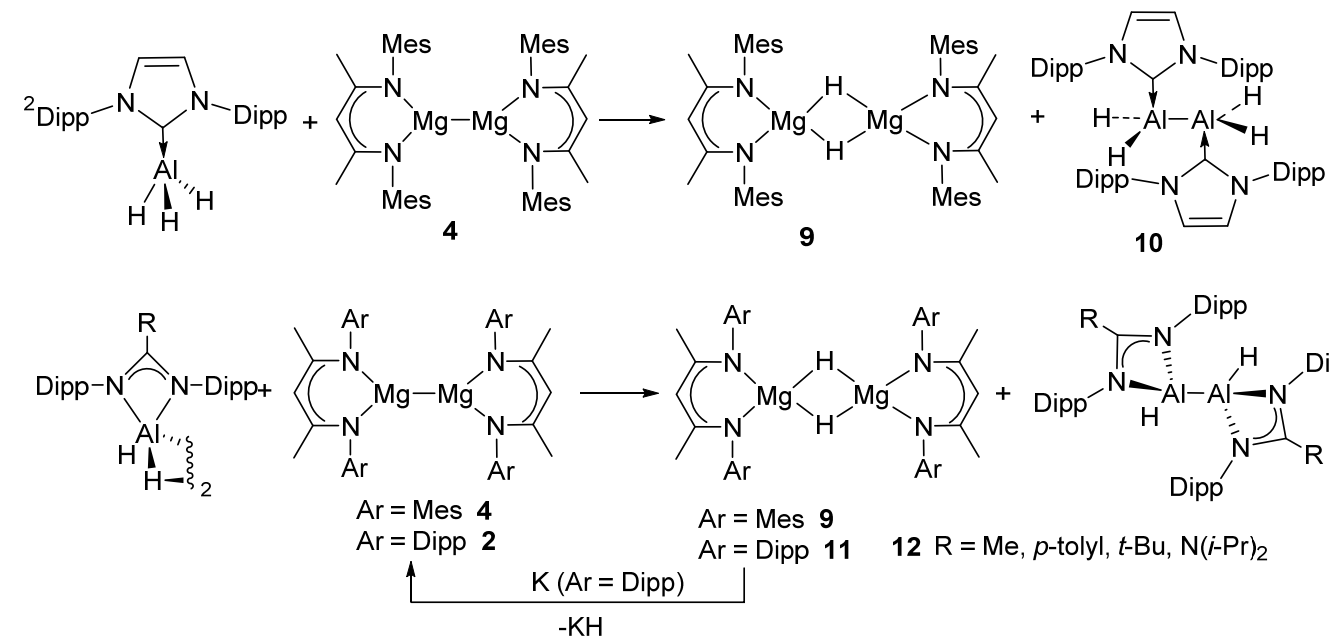

图式 3 化合物 $\mathbf{1 0}$ 和 $\mathbf{1 2}$ 的合成

Scheme 3 Synthesis of complexes 10 and $\mathbf{1 2}$ 
计算表明，上述生成 10 (和 9)的反应在室温下轻微放热 $(\Delta G=-4.0 \mathrm{kcal} / \mathrm{mol})$, 所以反应可能是可逆的. 虽然这 在实际中没有被证实, 但较大空间位阻的氢化物 11 可 由钾还原脱氢，再生得到一价镁化合物 2.

前面提到一价镁化合物很难与氢气反应, 然而温室 下二氧化碳却能被一价镁 $\left[\left\{\left({ }^{\mathrm{Dipp}} \mathrm{Nacnac}\right) \mathrm{Mg}\right\}_{2}\right]$ 还原 (Scheme 4). 该过程可能经过两步反应, 第一步是二氧 化碳与一价镁反应, 脱氧得到氧桥连中间体 13 (伴随着 副产物一氧化碳的释放), 反应中间体可以通过 $\mathrm{N}_{2} \mathrm{O}$ 与 一价镁化合物反应证实, 伴随着氮气的释放. 第二步是 中间体 13 继续与第二份二氧化碳分子反应得到主要产 物即碳酸酯镁金属络合物 14, 同时生成少量的副产物 二价乙二酸镁络合物 $\mathbf{1 5}^{[40,41]}$.

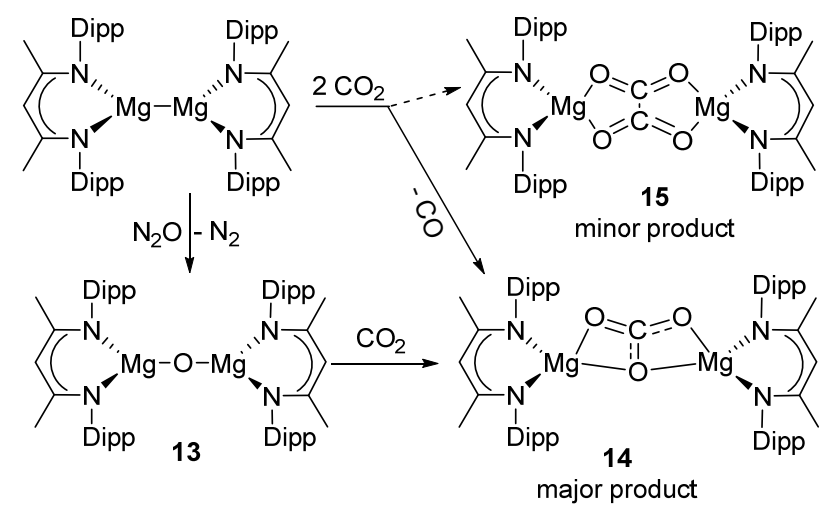

图式 4 化合物 14 的合成

Scheme 4 Synthesis of complexes 14

\subsection{2 一价镁化合物在 14 主族中的应用}

迄今为止，一价镁化合物在无机和金属有机合成中 最成功之处是作为还原剂制备了众多低氧化态 14 族化 合物. 最初的研究是低价锗配合物的合成, 这主要源于 早先 Robinson 等 ${ }^{[42]}$ 报道用 $\mathrm{KC}_{8}$ 还原卡宾硅化合物 $\left[(\mathrm{IPr}) \mathrm{SiCl}_{4}\right]$ 可以得到零价硅化物, 即 $[(\mathrm{IPr}) \mathrm{Si}=\mathrm{Si}(\mathrm{IPr})]$ $\left[\mathrm{IPr}=: \mathrm{C}\{\mathrm{N}(\mathrm{Dipp}) \mathrm{C}(\mathrm{H})\}_{2}\right]$. 这一发现导致了一系列后续 报道, 制备了众多其它卡宾配位的低价(零价或 +1 价) 主族金属化合物 ${ }^{[43 \sim 55}$. 然而 Jones 等试图通过金属钠或 $\mathrm{KC}_{8}$ 还原相应的锗化合物 $\left[(\mathrm{IPr}) \mathrm{GeCl}_{2}\right]$, 只生成了金属元 素锗 $\mathrm{Ge}$ 和卡宾原配体. 与此相反, 当上述反应在室温 下用等物质的量的一价镁化合物 2 或 4 作为还原剂时, 可以成功分离出目标产物, 即卡宾配位的零价锗化物 $16^{[46]}$, 虽然产率有点低(Eq. 2). 有趣的是, 当用 50 $\mathrm{mol} \%$ 的一价镁化合物反应时，推测的中间体 $\left[(\mathrm{IPr}) \mathrm{Ge}^{\mathrm{I}}(\mathrm{Cl})(\mathrm{Ge})^{\mathrm{I}}(\mathrm{Cl})(\mathrm{IPr})\right]$ 并没有得到, 最终得到化合 物 16 和过量未反应的原料 $\left[(\mathrm{IPr}) \mathrm{GeCl}_{2}\right]$. 反应产生的副 产物二价镁氯化物 $\left[\mathrm{LMg}(\mu-\mathrm{Cl})_{2} \mathrm{MgL}\right] \quad\left(\mathrm{L}={ }^{\mathrm{Dipp}} \mathrm{Nacnac}\right.$, ${ }^{\mathrm{Mes} N a c n a c)}$ 在乙醚中溶解性差, 所以容易从产物 16 中分
离出. 随后他们用同样的方法合成出类似的卡宾络合一 价锡化合物 $\mathbf{1 7}{ }^{[47]}$.

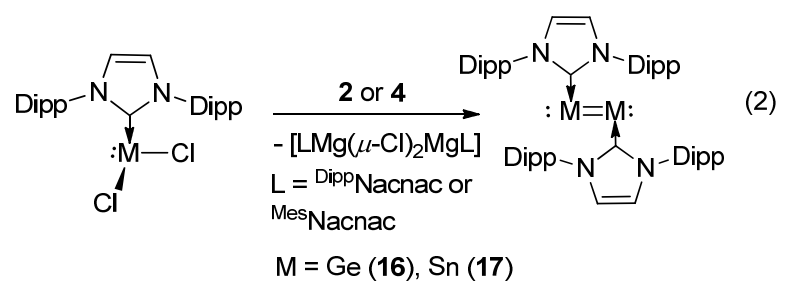

一价镁化合物在合成一系列单聚或二聚体 14 族一 价化合物是比碱金属更为有效的还原剂. 例如, 在 2006 年 Jones 等 ${ }^{[48}$ 报道了用金属钾还原二价锗化合物 [ $\left\{\mathrm{k}^{2}-\right.$ $\left.N, N^{\prime}-\mathrm{RC}(\mathrm{NDipp})_{2}\right\} \mathrm{GeCl}$ 制备出第一个脒基或胍基螯合 的一价锗化合物 $\left[\left\{\left[\mathrm{k}^{2}-N, N^{\prime}-\mathrm{RC}(\mathrm{NDipp})_{2}\right] \mathrm{Ge}\right\}_{2}\right](\mathrm{R}=t-\mathrm{Bu}$, $\left.\mathrm{N}\left(\mathrm{C}_{6} \mathrm{H}_{11}\right)_{2}\right)$. 几年后, Roesky 等 ${ }^{[49,50]}$ 用同样的方法合成结 构类似的一价锗和一价硅化合物 $\left[\left\{\left[k^{2}-N, N^{\prime}-\mathrm{PhC}\right.\right.\right.$ $\left.\left.\left.(\mathrm{NBu}-t)_{2}\right] \mathrm{E}\right\}_{2}\right](\mathrm{E}=\mathrm{Si}, \mathrm{Ge})$. Roesky 等 ${ }^{[51]}$ 合成的一价硅化 合物被证明可以高效活化小分子和不饱和有机底物, 然 而上述反应都存在产率较低的缺点. 为了进一步扩大在 这方面的研究进展，Jones 等 ${ }^{[52]}$ 用一价镁化合物替代金 属钾和 $\mathrm{KC}_{8}$ 作为还原剂, 将缕基配位前体 $\left[\left\{\mathrm{k}^{2}-N, N^{N}-\right.\right.$ $\left.\left.\left(\mathrm{C}_{6} \mathrm{H}_{4}(\mathrm{Bu}-t)-4\right) \mathrm{C}(\mathrm{NDipp})_{2}\right\} \mathrm{SiCl}_{3}\right]$ 或 $\left[\left\{\mathrm{k}^{2}-N, N^{\prime}-\left(\mathrm{C}_{6} \mathrm{H}_{4}(\mathrm{Bu}-t)-\right.\right.\right.$ 4) $\left.\left.\mathrm{C}(\mathrm{NDipp})_{2}\right\} \mathrm{ECl}\right](\mathrm{E}=\mathrm{Ge}, \mathrm{Sn})$ 分别和 1.5 或 0.5 equiv. 的 [\{ $\left.\left.\left({ }^{\mathrm{Mes}} \mathrm{Nacnac}\right) \mathrm{Mg}\right\}_{2}\right](4)$ 反应, 可以高产率得到相应的 一价硅和一价锗化合物 $18(91 \%)$ 和 $19(71 \%)$. 虽然相应 的一价锡化合物 20 的产率只有 $36 \%$, 但是之前没有类 似的报道(Eq. 3). 值得注意的是为了与一价镁比较, 金 属锂、萗基锂或葱镁分别还原 $\left[\left\{\mathrm{k}^{2}-N, N^{\prime}-\left(\mathrm{C}_{6} \mathrm{H}_{4}(\mathrm{Bu}-t)-\right.\right.\right.$ 4)C(NDipp $\left.\left.)_{2}\right\} \mathrm{SiCl}_{3}\right]$, 产率都比较低且产物很难提纯.

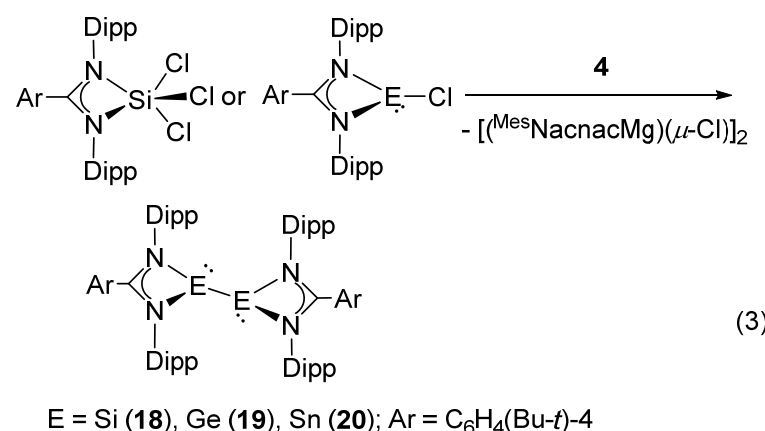

随后他们将一价镁 $\left[\left\{\left({ }^{\mathrm{Mes}} \mathrm{Nacnac}\right) \mathrm{Mg}\right\}_{2}\right]$ 作为还原剂 延伸到大空间位阻的单齿氨基配位的一价锗和一价锡 化合物 21 23 的合成中(Eq. 4) ${ }^{[53 \sim 55]}$. 例如尝试通过碱 金属或 $\mathrm{KC}_{8}$ 还原前体 $[\mathrm{LGeCl}]\left[\mathrm{L}=\mathrm{N}\left(\mathrm{Ar}^{*}\right)\left(\mathrm{SiMe}_{3}\right), \mathrm{Ar}^{*}=\right.$ $\left.\mathrm{C}_{6} \mathrm{H}_{2} \mathrm{Me}\left\{\mathrm{C}(\mathrm{H}) \mathrm{Ph}_{2}\right\}_{2}-4,2,6\right]$, 导致过度还原或产率非常低, 而一价镁 4 作为还原剂产率相对较高. 另外合成的一价 
锗化合物 21 非常活泼的, 在液态或甚至固态, 温度一 $10{ }^{\circ} \mathrm{C}$ 下也能和氢气反应得到混价的锗氢化物 $\left[\operatorname{LGeGe}(\mathrm{H})_{2} \mathrm{~L}\right]$, 其相应的氢化物非常高效催化一系列醛 和酮的硼氢化反应 ${ }^{[56]}$.

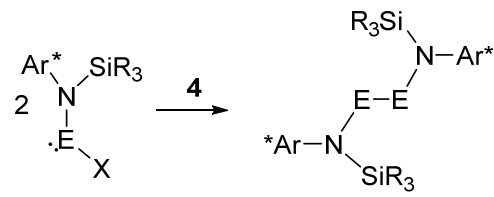

$\mathrm{Ar}^{*}=4,2,6-\mathrm{Me}\left\{\mathrm{C}(\mathrm{H}) \mathrm{Ph}_{2}\right\}_{2} \mathrm{C}_{6} \mathrm{H}_{2}, \mathrm{R}=\mathrm{Me}, \mathrm{X}=\mathrm{Cl}, \mathrm{E}=\mathrm{Ge}(21)$; $\mathrm{Ar}^{*}=4,2,6-i-\operatorname{Pr}\left\{\mathrm{C}(\mathrm{H}) \mathrm{Ph}_{2}\right\}_{2} \mathrm{C}_{6} \mathrm{H}_{2}, \mathrm{R}=i-\mathrm{Pr}, \mathrm{X}=\mathrm{Cl}, \mathrm{E}=\mathrm{Ge}(22)$; $\mathrm{X}=\mathrm{Br}, \mathrm{E}=\mathrm{Sn}(\mathbf{2 3})$

一价镁还原反应得到的上述一价锗化合物，可能通 过自由基的中间体. 由于之前没有类似自由基被分离的 报道, Jones 等 ${ }^{[57]}$ 用一价镁还原大空间位阻的 2,6-二异丙 基取代 $\beta$-二亚胺配位的二价锗前体，成功合成稳定的一 价锗自由基 24, 产率为 38\% (Scheme 5). 该自由基通过 $E P R / E N D O R$ 光谱和 $X$ 射线单晶衍射等表征, 并能与氯 化剂 $\mathrm{C}_{2} \mathrm{Cl}_{6}$ 反应, 定量地再生二价锗前体. 值得注意的 是，虽然二价锗前体和碱金属还原没有得到化合物 24, 但其与荎基钠的 THF 溶液反应，与一价镁化合物 $\mathbf{4}$ 为还 原剂相比较, 产率几乎相同. 但当空间位阻稍小的 $2,4,6$-三甲基取代的 $\beta$-二亚胺配位的锗氯化物与一价镁 4 反应时, 不能得到相应的一价锗自由基, 而分离出配 体 C- $\mathrm{N}$ 键断裂的 Ge-Mg 双金属化合物 25; 相应的铅化 合物用一价镁还原只得到分解的金属铅和二聚物等副 产物. 只有相应的二价锡氯化物和 $50 \mathrm{~mol} \%$ 的一价镁 4 反应，以中等产率 $(55 \%)$ 分离出第一个 $\beta$-二亚胺配位的 一价锡化合物 $\mathbf{2 6}^{[58]}$.

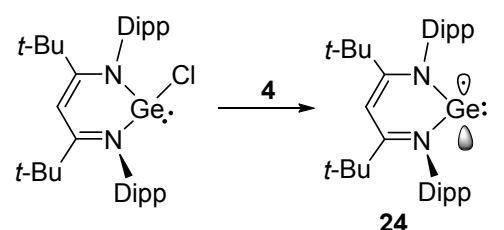

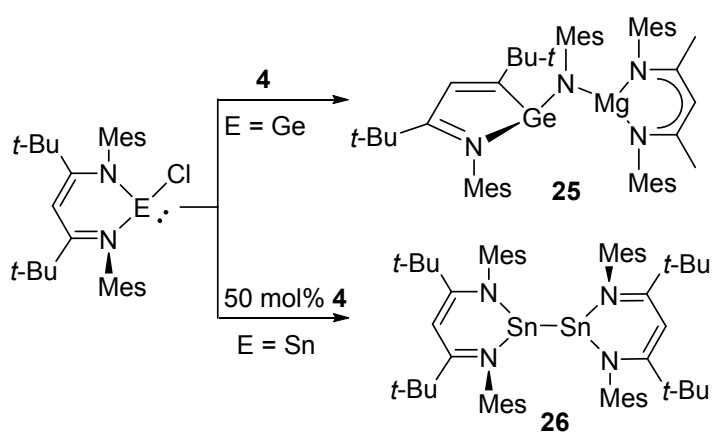

图式 5 化合物 24 26 的合成

Scheme 5 Synthesis of complexes 24 26

2011 年 Driess 等 ${ }^{[59]}$ 报道使用一价镁化合物作为还 原剂可以制备叶立德稳定碳环的硅烯(silylene) 27. 起初 硅的二溴化物前体和 2 equiv.的 $\mathrm{KC}_{8}$ 在 THF 中反应，但 该反应不能完全结束, 且产物随着反应时间的延长, 逐 渐分解, 副产物逐渐增多, 这可能由于产物在 THF 溶液 中不稳定. 如果用一价镁 4 作为还原剂, 反应选择性非 常好, 且最终产物在溶剂苯中和室温下可以稳定数月. 2012 年 Power 等 ${ }^{[60]}$ 将一价镁 $\mathbf{4}$ 和含有三苯基双硫配体 硅溴化物反应，合成出稳定的非环二配位的硅烯 28 (Scheme 6).

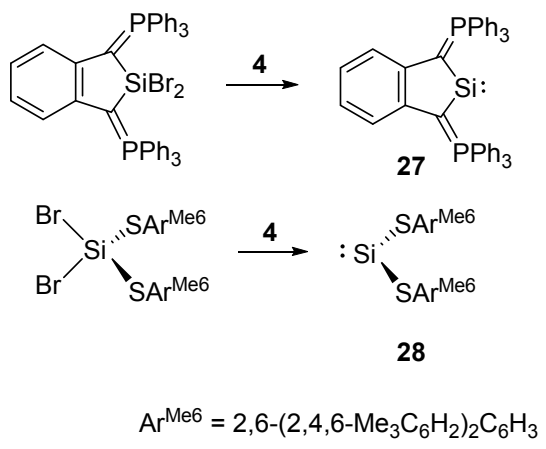

图式 6 化合物 $\mathbf{2 7}$ 和 28 的合成

Scheme 6 Synthesis of complexes 27 and 28

\subsection{3一价镁化合物在过渡金属中的应用}

一价镁化合物也可以用于低价过渡金属化合物的 合成中, 例如, 2012 年 Jones 等 ${ }^{[61]}$ 将胍基配位的铁溴化 物与等物质的量一价镁 $\mathbf{4}$ 反应得到胍基配位的深红棕色 一价铁化合物 29, 产率可以达到 57\% (Scheme 7); 而用 传统的还原剂镁或钾, 不能分离出目标产物, 而产生了 众多难以处理的混合物. 空间位阻较小的叔丁基取代的 胍基配位的铁溴化物与一价镁虽然产生了相应的一价
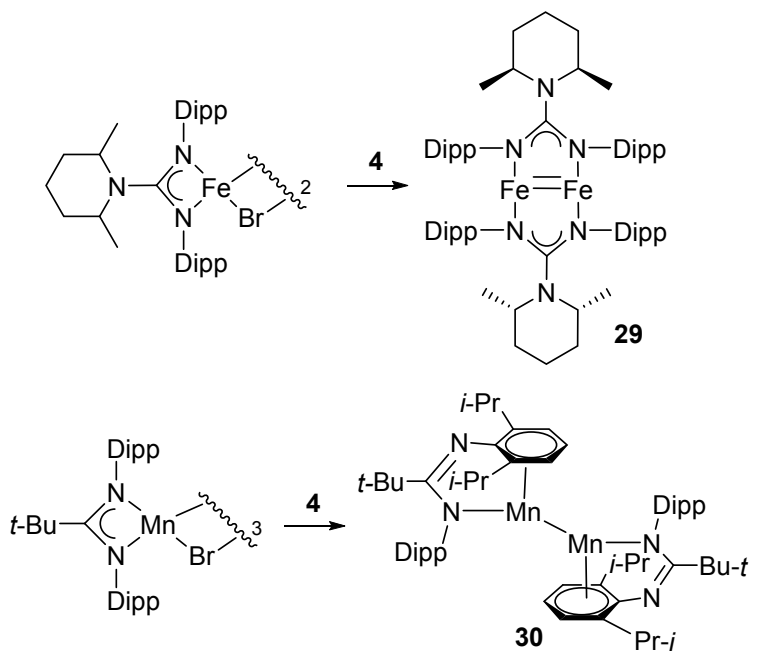

图式 7 化合物 $\mathbf{2 9}$ 和 $\mathbf{3 0}$ 的合成

Scheme 7 Synthesis of complexes 29 and $\mathbf{3 0}$ 
铁化合物, 但产物在溶液中非常容易发生分解, 生成其 它副产物. 同样的一价镁也可以应用于脒基配位的一价 锰的合成, 前体锰的三溴化物与 1.5 equiv.一价镁 $\mathbf{4}$ 反应 得到深红绿的一价锰化合物 $\mathbf{3 0}$, 有趣的是锰原子由前 体中的 $\mathrm{N}, \mathrm{N}$ 配位变为 $\mathrm{N}$ 和一个苯环配位.

之前 Jones 等 ${ }^{[2]}$ 报道大空间位阻的单齿氨基配体也 可以制备低价锰化合物, 空间位阻稍小的甲基取代的氨 基锰的溴化物前体与一价镁 $\mathbf{4}$ 反应，得到对称的二配位 的一价锰化合物 31, 而空间位阻稍大的异丙基取代的 氨基锰的溴化物前体与一价镁 $\mathbf{4}$ 反应, 则分离出 $\mathrm{Mn}-\mathrm{Mg}$ 双金属化合物 32, 其中二配位的锰为零价, 这个化合物 可以作为 “无机格氏试剂” 合成其它双金属化合物 (Scheme 8).

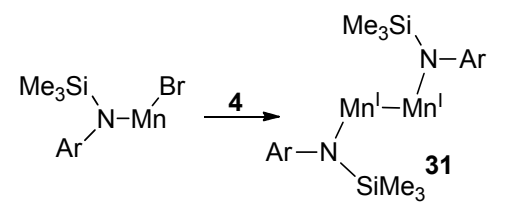

$\mathrm{Ar}=4,2,6-\mathrm{Me}\left\{\mathrm{C}(\mathrm{H}) \mathrm{Ph}_{2}\right\}_{2} \mathrm{C}_{6} \mathrm{H}_{2}$

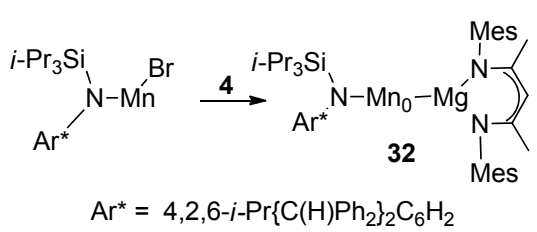

图式 8 化合物 31 和 $\mathbf{3 2}$ 的合成

Scheme 8 Synthesis of complexes $\mathbf{3 1}$ and $\mathbf{3 2}$

2013 年 Braunschweig 等 ${ }^{[63]}$ 用一价镁 4 作为还原剂 制备首个零价铂稳定的嗍烯络合物 33 (Scheme 9). 我 们 ${ }^{[64]}$ 也将一价镁应用于双金属簇合物的合成中, 例如 零价铂 $\mathrm{Pt}\left(\mathrm{PCy}_{3}\right)_{2}$ 与 14 族金属二氯化物反应得到结构新 颖的 MOLP (metal only Lewis pair)金属配合物, 只有相 应的锡化合物能与一价镁 4 反应, 分离出含 7 个铂金属 和 8 个锡金属的黑色双金属簇合物 $\mathbf{3 4}$, 而类似锗和铅化 合物只分离出起始原料零价铂 $\operatorname{Pt}\left(\mathrm{PCy}_{3}\right)_{2}$.

近年来由于能源短缺问题不断加重, 氨硼烷 $\left(\mathrm{NH}_{3} \bullet \mathrm{BH}_{3}\right)$ 作为含氢量高达 $19.6 \mathrm{wt} \%$ 的储氢材料被广泛 研究, 然而其存在脱氢温度较高 (110 ${ }^{\circ} \mathrm{C}$ 以上) 等问题. 一价镁化合物 2 和氨硼烷在 $-60{ }^{\circ} \mathrm{C}$ 就可释放出氢气, 同时生成氨基嗍镁的络合物 $\mathbf{3 5}$ (Eq. 5) ${ }^{[65]}$.

\section{2 一价镁化合物的小分子反应性}

镁金属化合物在有机合成方面的应用已经超过一 百多年, 毫无疑问应用最多是格氏试剂 ${ }^{[66,67]}$. 由于格氏 试剂中金属镁直接和碳链相连, 极化作用使邻近镁原子 的碳原子呈负电性, 使 $\mathrm{C}-\mathrm{Mg}$ 键具有反应活性. 它们参 加的一些反应比如频哪醇偶联, 被认为是镁化合物通过
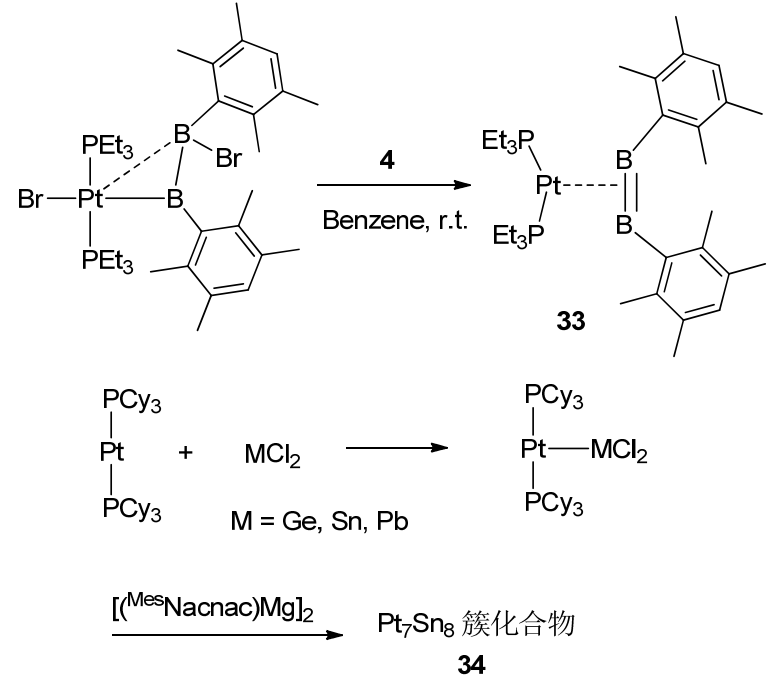

图式 9 化合物 33 和 34 的合成

Scheme 9 Synthesis of complexes $\mathbf{3 3}$ and $\mathbf{3 4}$

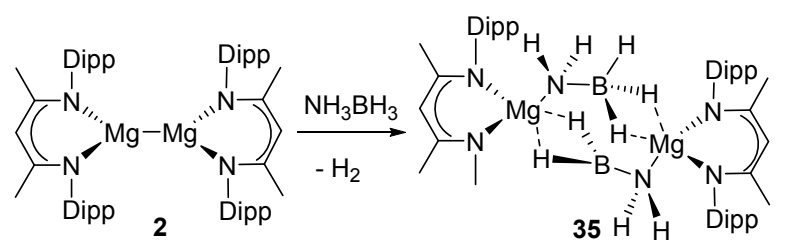

单电子转移到反应物. 同样, $\mathrm{Mg} / \mathrm{MgX}_{2}(\mathrm{X}=\mathrm{Br}$ 或 I $)$ 混合 物也被应用于许多涉及单电子还原的有机反应 ${ }^{[68]}$, 它 们的还原能力推测可能来源于混合物中微量的一价镁 化合物 $\cdot M g X$ 或 $\mathrm{XMgMgX}$. 目前, 有机合成中作为单电 子还原剂应用最广的不是镁金属化合物，而是已经商业 化的碘化钐 $\mathrm{SmI}_{2}$, 然而在很多反应中还原剂为 $\mathrm{SmI}_{2}$ 的 反应选择性难以控制, 所以有机化学研究者希望获得更 多可选择的还原剂. 而上述合成的室温稳定一价镁化合 物有望克服二碘化钐的化学选择性较差的缺点.

为了确定一价镁化合物在这方面是否合适? Stasch 和 Jones 等 ${ }^{[69]}$ 检验了它们对多种不饱和有机化合物的反 应活性. 在目前研究的所有反应中，一价镁化合物可作 为双中心双电子的还原剂. 例如偶氮苯 $(\mathrm{PhN}=\mathrm{NPh})$ 、碳 化二亚胺 $(\mathrm{CyN}=\mathrm{C}=\mathrm{NCy})$ 、环辛四烯 $(\mathrm{COT})$ 和葱等嵌入 一价镁化合物中的 $\mathrm{Mg}-\mathrm{Mg}$ 键中, 形成一系列结构新颖 的化合物 36 39, 产率都比较高(Scheme 10). 这表明一 价镁化合物可广泛地应用于有机合成. 例如, 虽然含有 两个被还原偶氮苯的化合物很多，但去芳环化结构的化 合物 36 还是前所未有的. 此外化合物 37 是仅有两个已 报道的缕基美化物中的第一个，另一个是由一价镁 [\{(Priso) $\left.M g\}_{2}\right](1)$ 和碳化二亚胺合成的 $[$ (Priso) $\mathrm{Mg}\{\mu$ (NDipp) $\left.{ }_{2} \mathrm{C}\right\} \mathrm{Mg}$ (Priso)], 这是 $\mathbf{1}$ 作为还原剂唯一合成的化 合物. 化合物 $\mathbf{3 8}$ 是通过晶体学表征的镁- COT 化合物之 一，高溶解度的化合物 39 能取代溶解性较差的 

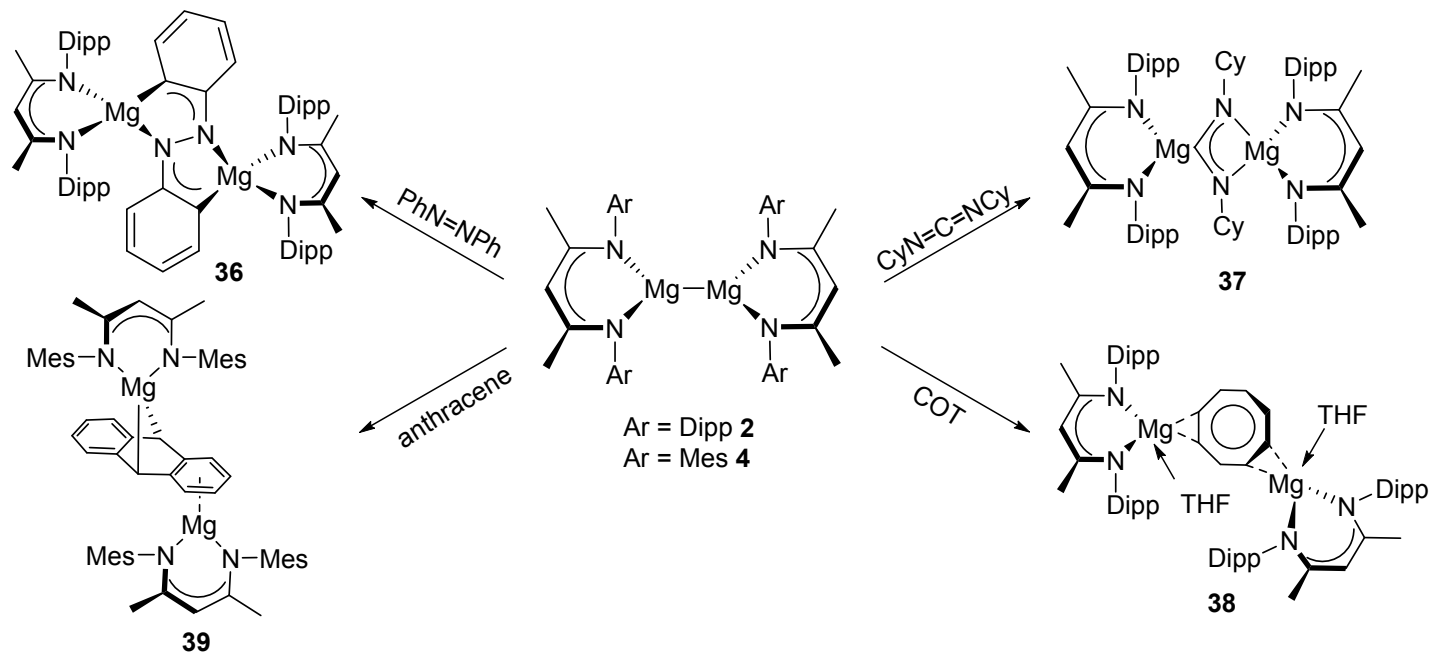

图式 10 化合物 36 39 的合成

Scheme 10 Synthesis of complexes 36 39

$\left[(\mathrm{THF})_{3} \mathrm{Mg}(\right.$ anthracene $\left.)\right]$ 作为还原剂.

一价镁化合物也可用于不饱和有机底物的 $\mathrm{N}-\mathrm{N}$ 和 $\mathrm{C}-\mathrm{C}$ 键的偶联反应, 如金刚烷叠氮化物 $\left(\mathrm{AdN}_{3}\right)$ 与一价 镁 2 发生首个由主族金属引发叠氮化合物的 $\mathrm{N}-\mathrm{N}$ 偶联 反应, 生成不常见的化合物 $\mathbf{4 0}$, 其含有 6 个共价氮, 可 用于合成氮杂环化合物或作为高能材料的前体. 一价镁 化合物与异氧酸酯、腈化物和异腈化物都可发生 $\mathrm{C}-\mathrm{C}$
偶联反应，生成一系列结构新颖的新化合物，一价镁中 $\beta$-二亚胺配体结构对最终产物的生成有显著的影响, 结 构的细微改变 (由 ${ }^{\mathrm{Dipp}} \mathrm{Nacnac}$ 转变 ${ }^{\mathrm{Mes}} \mathrm{Nacnac}$ ) 导致了不同 反应产物的生成. 例如, 一价镁 $\mathbf{2}$ 和 $\mathbf{4}$ 与叔丁基异氰酸 酯 $(t-\mathrm{BuNCO})$ 反应生成不同结构的乙二酰二胺镁金属化 合物 41 和 42 (Scheme 11)，一价镁 8 也可发生类似的反 应. $\left[\left\{\left({ }^{\mathrm{Mes}} \mathrm{Nacnac}\right) \mathrm{Mg}\right\}_{2}\right]$ 和异腈化物 $t$-BuNC 和 XylNC,

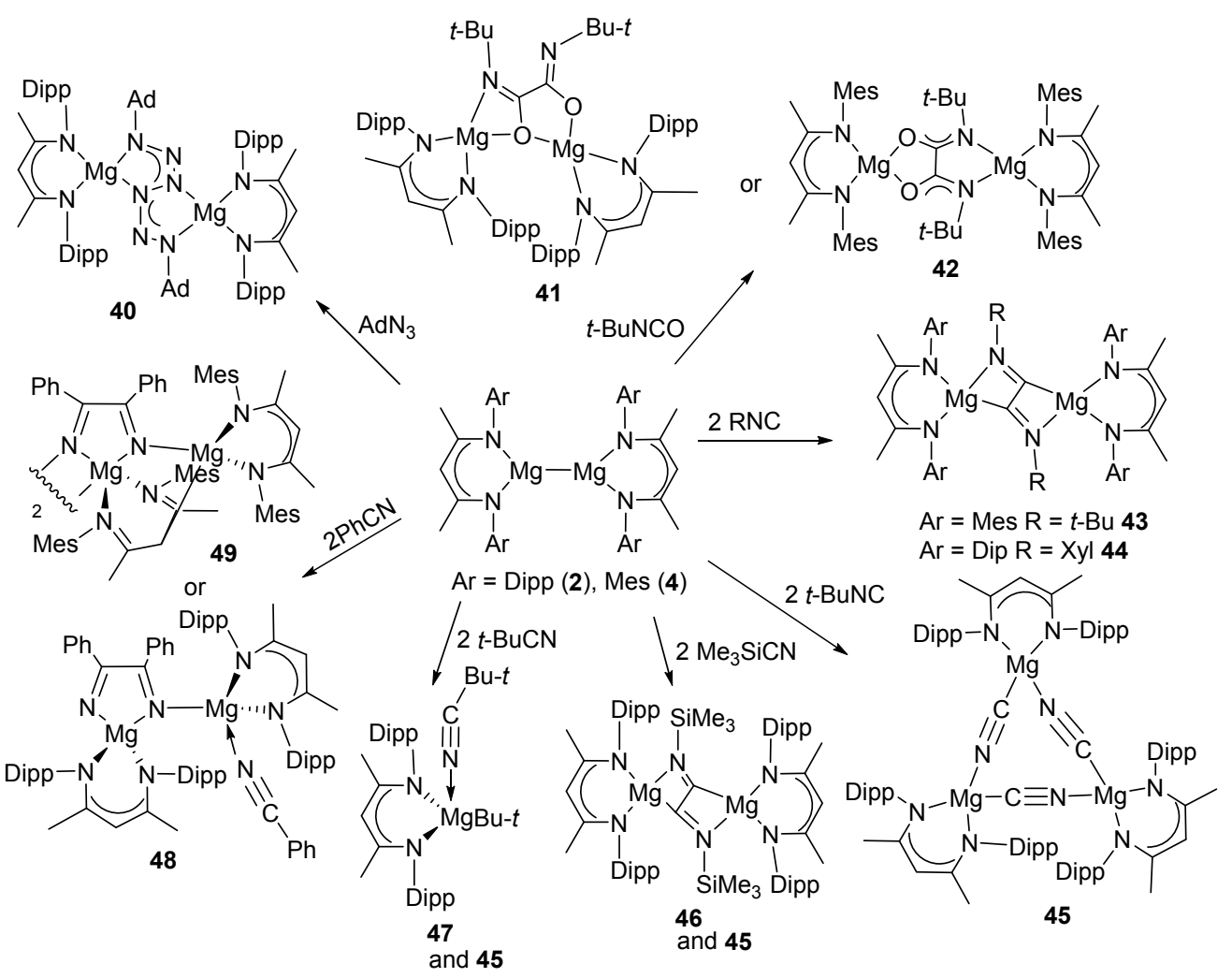

图式 11 化合物 $40 \sim 49$ 的合成

Scheme 11 Synthesis of complexes $40 \sim 49$ 
$\left(\mathrm{Xyl}=2,6-\mathrm{Me}_{2} \mathrm{C}_{6} \mathrm{H}_{3}\right)$ 反应生成紫色的 $\mathrm{C}-\mathrm{C}$ 偶联产物 43 和 44, 而用空间位阻稍大的 $\left[\left\{\left({ }^{\mathrm{Dipp}} \mathrm{Nacnac}\right) \mathrm{Mg}\right\}_{2}\right]$ 则得到 了完全不同的结果, $\mathrm{C}-\mathrm{N}$ 键断裂生成三聚物 [\{( $\left.\left.\left.{ }^{\text {Dipp }} \mathrm{Nacnac}\right) \mathrm{Mg}(\mu-\mathrm{CN})\right\}_{3}\right](45) ;$ 一价镁 2 与三甲基硅腈 化物 $\left(\mathrm{Me}_{3} \mathrm{SiCN}\right)$ 反应生成 $\mathrm{C}-\mathrm{C}$ 偶联产物 46 和三聚物 45 , 而与叔丁基腈化物 $(t-\mathrm{BuCN})$ 反应只生成单核的叔丁基 镁金属化合物 47 和三聚物 45. [ [ $\left.\left({ }^{\mathrm{Dipp}} \mathrm{Nacnac}\right) \mathrm{Mg}_{2}\right]$ 与苄 腈 $(\mathrm{PhCN})$ 反应导致了一次 $\mathrm{C}-\mathrm{C}$ 偶联产物 48 和一份子 未反应的苄腈, $\left[\left\{\left({ }^{\mathrm{Mes}} \mathrm{Nacnac}\right) \mathrm{Mg}\right\}_{2}\right]$ 由于空间位阻稍小则 发生了两次 $\mathrm{C}-\mathrm{C}$ 偶联反应 $49^{[70]}$.

另外, 二苯甲酮与一价镁还原后再加入 4-二甲氨基 吡啶(DMAP), 得到高产率、稳定的、蓝紫色的镁化合物 50, 这是第一个晶体表征的镁游离羰基化合物. 一价镁 化合物 2 分别和强的路易斯碱四氢呋喃(THF)、1,4-二氧 六环(dioxane)、4-二甲氨基吡啶(DMAP)或 4-叔丁基吡啶 $(t$-BuPy) 反应, 得到相应的一价镁的路易斯碱加合物 51 54; 而一价镁化合物 4 只能与 THF 和 DMAP 反应 得到化合物 55 和 56 (Scheme 12) ${ }^{[71,72]}$.

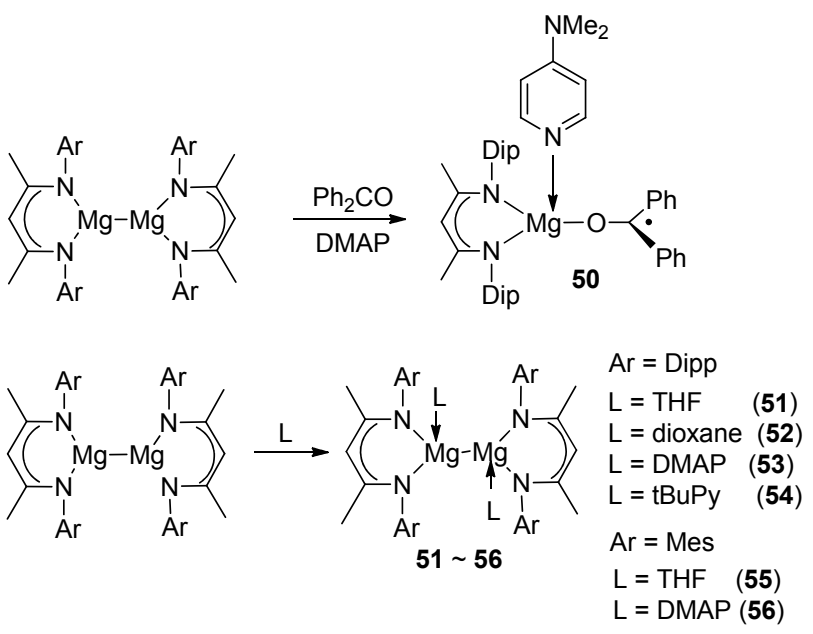

图式 12 化合物 $50 \sim 56$ 的合成

Scheme 12 Synthesis of complexes $50 \sim 56$

\section{3 结论}

迄今为止，一系列大空间位阻有机配体稳定的一价 镁金属化合物已经成功合成. 相对于传统的还原剂, 它 们可作为可溶的、化学计量可控的、选择性的和安全的 还原剂应用于众多的无机和有机合成中, 其作为催化剂 在无机和有机反应中将有着更广阔的应用前景. 有望在 不远的将来代替昂贵的过渡金属催化剂应用于实验室 及工业合成中, 因此深入探索新型一价镁金属化合物的 合成及其反应性能值得引起人们的注意和进一步的研 究.

\section{References}

[1] Cotton, F. A.; Curtis, N. F.; Harris, C. B.; Johnson, B. F. G.; Lippard, S. J.; Mague, J. T.; Robinson, W. R.; Wood, J. S. Science 1964, 145, 1305.

[2] Resa, I.; Carmona, E.; Gutierrez-P, E.; Monge, A. Science 2004 $305,1136$.

[3] Nguyen, T.; Sutton, A. D.; Brynda, M.; Fettinger, J. C.; Long, G. J.; Power, P. P. Science 2005, 310, 844.

[4] Fischer, R. C.; Power, P. P. Chem. Rev. 2010, 110, 3877.

[5] Power, P. P. Organometallics 2007, 26, 4362.

[6] Rivard, E.; Power, P. P. Inorg. Chem. 2007, 46, 10047.

[7] Pu, L.; Twamley, B.; Power, P. P. J. Am. Chem. Soc. 2000, 122, 3524.

[8] Stender, M.; Phillips, A. D.; Wright, R. J.; Power, P. P. Angew. Chem., Int. Ed. 2002, 41, 1785.

[9] Sekiguchi, A.; Kinjo, K.; Ichinohe, M. Science 2004, 305, 1755.

[10] Wiberg, N.; Niedermayer, W.; Fischer, G.; Noth, H.; Suter, M. Eur. J. Inorg. Chem. 2002, 5, 1066.

[11] Wang, X.; Andrews, L. J. Phys. Chem. A 2004, 108, 11511.

[12] Tague, T. J.; Andrews, L. J. Phys. Chem. 1994, 98, 8611.

[13] Green, S. P.; Jones, C.; Stasch, A. Science 2007, 318, 1754.

[14] Bonyhady, S. J.; Jones, C.; Nembenna, S.; Stasch, A.; Edwards, A. J.; McIntyre, G. J. Chem. Eur. J. 2010, 16, 938.

[15] Stasch, A.; Jones, C. Dalton. Trans. 2011, 40, 5659.

[16] Jones, C.; Stasch, A. Top. Organomet. Chem. 2013, 45, 73.

[17] Grirrane, A.; Resa, I.; Rodriguez, A.; Carmona, E.; Alvarez, E.; Gutierrez-P, E.; Monge, A.; Galindo, A.; Del, R. D.; Andersen, R. A. J. Am. Chem. Soc. 2007, 129, 693.

[18] Grirrane, A.; Resa, I.; Rodriguez, A.; Carmona, E. Coord. Chem. Rev. 2008, 252, 1532.

[19] Li, T.; Schulz, S.; Roesky, P. W. Chem. Soc. Rev. 2012, 41, 3759.

[20] Yang, X.; Yu, J.; Liu, Y.; Xie, Y.; Schaefer, H. F.; Linag, Y.; Wu, B. Chem. Commun. 2007, 43, 2363.

[21] Yu, J.; Yang, X.; Liu, Y.; Pu, Z.; Li, Q.; Xie, Y.; Schaefer, H. F.; Wu, B. Organometallics 2008, 27, 5800 .

[22] Yang, P.; Yang, X.; Yu, J.; Liu, Y.; Zhang, C.; Deng, Y.; Wu, B. Dalton. Trans. 2009, 38, 5773.

[23] Liu, Y.; Li, S.; Yang, X.; Yang, P.; Gao, J.; Xia, Y.; Wu, B. Organometallics 2009, 28, 5270 .

[24] Gao, J.; Li, S.; Zhao, Y.; Wu, B.; Yang, X. Organometallics 2012, 31, 2978.

[25] Luehl, A.; Nayek, H. P.; Blechert, S.; Roesky, P. W. Chem. Commun. 2011, 47, 8280

[26] Liu, Y.; Li, S.; Yang, X.; Yang, P.; Wu, B. J. Am. Chem. Soc. 2009, 131,4210 .

[27] Gao, J.; Liu, Y.; Zhao, Y.; Yang, X.; Sui, Y. Organometallics 2011, 30,6071 .

[28] Stasch, A. Angew. Chem., Int. Ed. 2014, 53, 10200

[29] Kruczyński, T.; Pushkarevsky, N.; Henke, P.; Koeppe, R.; Baum, E.; Konchenko, S.; Pikies, J.; Schnoeckel, H. Angew. Chem., Int. Ed. 2012, 51, 9025.

[30] Pankewitz, T.; Klopper, W.; Henke, P.; Schnoeckel, H. Eur. J. Inorg. Chem. 2008, 31, 4879 .

[31] Köppe, R.; Henke, P.; Schnöckel, H. Angew. Chem., Int. Ed. 2008, 47,8740 .

[32] Nicolaou, K. C.; Ellery, S. P.; Chen, J. S. Angew. Chem., Int. Ed. 2009, $48,7140$.

[33] Kagan, H. B. Tetrahedron 2003, 59, 10351.

[34] Connelly, N. G.; Geiger, W. E. Chem. Rev. 1996, 96, 877.

[35] Power, P. P. Nature 2010, 463, 171.

[36] Power, P. P. Acc. Chem. Res. 2011, 44, 627. 
[37] Asay, M.; Jones, C.; Driess, M. Chem. Rev. 2011, 111, 354.

[38] Bonyhady, S. J.; Collis, D.; Frenking, G.; Holzmann, N.; Jones, C.; Stasch, A. Nat. Chem. 2010, 2, 865.

[39] Weng, X.; Andrews, L.; Tam, S.; DeRose, M. E.; Fajardo, M. E. J. Am. Chem. Soc. 2003, 125, 9218.

[40] Lalrempuia, R.; Stasch, A.; Jones, C. Chem. Sci. 2013, 4, 4383.

[41] Kefalidis, C. E.; Stasch, A.; Jones, C.; Maron, L. Chem. Commun. 2014, 50, 12318.

[42] Wang, Y.; Xie, Y.; Wei, P.; King, R. B.; Schaefer, H. F.; Schleyer, P. R.; Robinson, G. H. Science 2008, 321, 1069.

[43] Wang, Y.; Robinson, G. H. Chem. Commun. 2009, 45, 5201.

[44] Wolf, R.; Uhl, W. Angew. Chem., Int. Ed. 2009, $48,6774$.

[45] Dyker, C. A.; Bertrand, G. Science 2008, 321, 1050.

[46] Sidiropoulos, A.; Jones, C.; Stasch, A.; Klein, S.; Frenking, G. Angew. Chem., Int. Ed. 2009, 48, 9701.

[47] Jones, C.; Sidiropoulos, A.; Holzmann, N.; Frenking, G.; Stasch, A. Chem. Commun. 2012, 48, 9855.

[48] Green, S. P.; Jones, C.; Junk, P. C.; Lippert, K. A.; Stasch, A. Chem. Commun. 2006, 42, 3978.

[49] Sen, S. S.; Jana, A.; Roesky, H. W.; Schulzke, C. Angew. Chem., Int. Ed. 2009, 48, 8536.

[50] Nagendran, S.; Sen, S. S.; Roesky, H. W.; Koley, D.; Grubmuller, H.; Pal, A.; Herbst-I, R. Organometallics 2008, 27, 5459.

[51] Sen, S. S.; Khan, S.; Samuel, P. P.; Roesky, H. W. Chem. Sci. 2012, 3,659 .

[52] Jones, C.; Bonyhady, S. J.; Holzmann, N.; Frenking, G.; Stasch, A. Inorg. Chem. 2011, 50, 12315.

[53] Li, J.; Schenk, C.; Goedecke, C.; Frenking, G.; Jones, C. J. Am. Chem. Soc. 2011, 133, 18622.

[54] Hadlington, T. J.; Hermann, M.; Li, J.; Frenking, G.; Jones, C. Angew. Chem., Int. Ed. 2013, 52, 10119.

[55] Hadlington, T. J.; Jones, C. Chem. Commun. 2014, 50, 2321.

[56] Hadlington, T. J.; Hermann, M.; Frenking, G.; Jones, C. J. Am. Chem. Soc. 2014, 36, 28.

[57] Woodul, W. D.; Carter, E.; Muller, R.; Richards, A. F.; Stasch, A.;
Kaupp, M.; Murphy, D. M.; Driess, M.; Jones, C. J. Am. Chem. Soc. 2011, 133, 10074.

[58] Choong, S. L.; Christian, S.; Stasch, A.; Dange, D.; Jones, C. Chem. Commun. 2012, 48, 2504.

[59] Asay, M.; Inoue, S.; Driess, M. Angew. Chem., Int. Ed. 2011, 50, 9589.

[60] Rekken, B. D.; Thomas, M.; Fettinger, J. C.; Tuononen, H. M.; Power, P. P. J. Am. Chem. Soc. 2012, 134, 6504.

[61] Fohlmeister, L.; Liu, S.; Schulten, C.; Moubaraki, B.; Stasch, A.; Cashion, J. D.; Murray, K. S.; Gagliardi, L.; Jones, C. Angew. Chem., Int. Ed. 2012, 51, 8294.

[62] Hicks, J.; Hoyer, C. E.; Moubaraki, B.; Manni, G. L.; Carter, E.; Murphy, D. M.; Murray, K. S.; Gagliardi, L.; Jones, C. J. Am. Chem. Soc. 2014, 136, 5283.

[63] Braunschweig, H.; Damme, A.; Dewhurst, R. D.; Vargas, A. Nat. Chem. 2013, 5, 115.

[64] Hupp, F.; Mengtao, M.; Kroll, F.; Jimenez-H, J. O. C.; Dewhurst, R. D.; Radacki, K.; Stasch, A.; Jones, C.; Braunschweig, H. Chem. Eur. J. 2014, 20, 16888.

[65] Jones, C.; Bonyhady, S. J.; Nembenna, S.; Stasch, A. Chem. Eur. J. 2012, 2012, 2596.

[66] Rappoport, Z.; Marek, I. The Chemistry of Organomagnesium Compounds, Part 1, Chichester, Wiley, 2008.

[67] Richey, H. G. Jr. Grignard Reagents, New Developments, Chichester, Wiley, 2000.

[68] Rausch, M. D.; McEwen, W. E.; Kleinberg, J. Chem. Rev. 1957, 57, 417.

[69] Bonyhady, S. J.; Green, S. P.; Jones, C.; Nembenna, S.; Stasch, A. Angew. Chem., Int. Ed. 2009, 48, 2973.

[70] Mengtao, M.; Stasch, A.; Jones, C. Chem. Eur. J. 2012, 18, 10669.

[71] Green, S. P.; Jones, C.; Stasch, A. Angew. Chem., Int. Ed. 2008, 47, 9079.

[72] Jones, C.; McDyre, L.; Murphy, D. M.; Stasch, A. Chem. Commun. 2010, 46, 1511. 